\title{
Le syndrome douloureux chronique pelvien chez I'homme. Quelle physiopathologie? Quel traitement?
}

\author{
Christian BAUDE \\ Hôpital Edouard Herriot, Lyon
}

\section{RESUME}

Que l'homme soit jeune ou âgé, la douleur pelvienne chronique a toujours interpellée le corps médical avec des problèmes d'évaluation et de prise en charge thérapeutique.

Sa prévalence est élevée et sous-estimée comme en témoigne les chiffres suivants : 4 à $8 \%$ de consultants en centre de douleur chronique, $15 \%$ de consultants en milieu urologique pour des symptômes de type prostatite chronique avec altération de la qualité de vie, 70000 cas de cystite chronique par an aux USA.

Les circonstances d'apparition sont multiples à savoir un contexte post-opératoire, un contexte post-traumatique majeur ou mineur ou post-infectieux, parfois sans étiologie particulière et souvent dans un contexte multifactoriel.

La physiopathologie est donc imprécise et mal connue, en effet environ seulement $5 \%$ des prostatites chroniques ont une origine bactérienne. Cependant toute stimulation quelle qu'elle soit active les voies de la douleur avec d'abord une inflammation neurogène, puis une sensibilisation centrale avec modification de la plasticité neuronale, et enfin une douleur chronique rebelle avec dysfonction organique.

Ce mécanisme est évoqué actuellement dans de nombreuses publications concernant les douleurs pelviennes chroniques post-opératoires et les cystites et prostatites chroniques rebelles. Au final, la physiopathologie de ces douleurs est probablement neurogène. En l'absence d'une stimulation, un syndrome canalaire sur le nerf pudendal peut être évoqué.

Le traitement de la douleur pelvienne chronique de l'homme peut être envisagé de la façon suivante : un traitement étiologique si possible, un traitement médical à visée neurogène (antidépresseurs tricycliques pour la douleur continue, anticonvulsivants pour la douleur intermittente, anti NMDA en cas d'échec), un traitement de la dysfonction organique, un bloc analgésique du nerf pudendal en cas de suspicion d'un syndrome canalaire et un traitement global du patient en raison de l'altération de la qualité de vie.
En conclusion, une meilleure approche physiopathologique de ces douleurs pelviennes chroniques permet d'améliorer ces patients difficiles.

Mots clés: douleur pelvienne chronique, prostatite chronique, nerf pudendal

\section{INTRODUCTION}

Qu'elle soit aiguë ou chronique, qu'elle atteigne l'homme jeune ou âgé, la douleur pelvi-périnéale a toujours interpellé le corps médical et paramédical avec des problèmes d'évaluation, de recherche diagnostique, d'exploration clinique et paraclinique, et de prise en charge thérapeutique $[2,3,5,13,17,29,34]$. Ces difficultés proviennent de plusieurs facteurs : un problème de définition, une grande dispersion des patients vers de nombreux spécialistes et une insuffisance très nette de formation médicale et para médicale conduisant à une réputation de prise en charge difficile et délicate.

En effet il existe un amalgame dans la littérature entre douleur pelvienne et douleur périnéale [28]. La douleur pelvienne est définie comme une douleur située dans la partie inférieure de l'abdomen, au dessus du perinée, au niveau des tissus et viscères pelviens. La douleur périnéale se définit comme une douleur située à la partie inférieure du bassin, au dessous du diaphragme pelvien des releveurs de l'anus (périnée antérieur et postérieur) [5]. II en découle une implication clinique, diagnostique et thérapeutique.

Par ailleurs, les patients sont dispersés vers de nombreux spécialistes (urologue, proctologue, neurologue, interniste, psychiatre, algologue) et cela nuit à une bonne prise

\section{Correspondance :}

Dr Christian BAUDE - Consultation et traitement de la douleur chronique, Pavillon P, Hôpital Edouard Herriot, Place d'Arsonval, 69437 Lyon cedex 03 - Tel 04.72.11.01.53 Fax 04.72.68.46.08 - Email christian.baude@chu-lyon.fr 
en charge. Le développement des consultations de la douleur chronique permet un regroupement de ces patients avec des avis pluridisciplinaires efficaces sur le plan de l'évaluation et du traitement, et ce malgré le fait que les douleurs pelvi-périnéales soient encore un sujet tabou.

Quant à la formation médicale et paramédicale, quelle lacune !! Quelques heures à la Faculté sur la douleur en général et sur la douleur pelvienne ; c'est encore plus restreint même lors de formation pour les médecins spécialistes.

Au terme de ces considérations l'incidence des douleurs pelvi-périnéales paraît sous-estimée avec une prise en charge à améliorer [28, 29, 34].

Quelle est l'épidémiologie des douleurs pelviennes chroniques chez l'homme ? Du fait de la dispersion de ces patients souffrant de douleurs pelviennes chroniques vers de nombreux spécialistes, d'importantes références bibliographiques spécialisées font état d'une prévalence élevée et sous-estimée $[2,3,5,8,13,18,22,24,28,30,34]$. Les points forts de ces références sont les suivantes:

- 4 à $8 \%$ de consultants en centre de douleur chronique [5] ;

- $50 \%$ de patients consultent en milieu proctologique pour une douleur périnéale chronique avec en moyenne $5 \%$ de douleurs d'allure neuropathique ;

- $15 \%$ d'hommes consultent en milieu urologique pour des symptômes de type prostatite chronique [22] ;

- $10 \%$ de formes douloureuses abdomino-pelviennes pour la pathologie myofasciale [28] et $60 \%$ de formes douloureuses pelviennes pour le colon irritable ;

- 5 à $10 \%$ de consultations en milieu urologique pour douleur testiculaire chronique [28] ;

- 13 à $33 \%$ d'abus sexuels avec douleur pelvienne chronique rebelle [23].

Parmi toutes les étiologies, les pathologies chroniques douloureuses les mieux documentées sont la prostatite chronique, la cystite interstitielle et la douleur testiculaire chronique.

1. La prostatite chronique $[8,10,13,15,16,17,22,24$, $26,34]$

Les troubles mictionnels sont souvent au premier plan avec dysurie, pollakiurie et brûlure, associés à des douleurs chroniques de topographie variable : périnéale, hypogastique irradiant parfois vers les organes génitaux externes, et aggravées par la position assise. L'origine est bactérienne dans $5 \%$ des cas seulement avec possibilité d'explication neuropathique par inflammation neurogène. Le diagnostic est échographique avec un traitement difficile (traitement neurogène ?) et de nombreux échecs thérapeutiques. Citons quelques chiffres :

- $25 \%$ des consultations d'urologie aux USA concernent la prostatite chronique;

- $12 \%$ des hommes âgés de 40 à 79 ans aux USA présentent des signes de prostatite chronique avec altération de la qualité de vie.
2. La cystite intersticielle et chronique $[10,18,19,25$, $30,34]$

Les symptômes associent pollakiurie, douleurs pelviennes, urgences mictionnelles et brûlures mictionnelles. La physiopathologie est mal connue (auto-immune, neurogéne, allergie ?) avec donc des difficultés thérapeutiques. Le diagnostic se fait par la clinique et par les biopsies vésicales.

C'est un probléme de santé public majeur, en effet :

- 70000 cas aux USA ;

- Prévalence entre $51 / 10000$ et $67 / 10000$ avec 9 femmes pour 1 homme ;

- Qualité de vie très altérée.

Mais finalement les douleurs pelviennes chroniques chez I'homme surviennent souvent dans des circonstances multiples mais précises. Les circonstances d'apparition habituelles sont :

- contexte post-opératoire pelvien précoce ou tardif ;

- contexte post-infectieux souvent tardif après une infection urinaire, une prostatite ou après une infection virale de type herpés par exemple ;

- contexte post-traumatique mineur ou majeur (traumatisme du bassin, du coccyx ou microtraumatismes répétés comme la pratique de certains sports ou la station assise prolongée);

- souvent un contexte mixte par association des circonstances précédentes ;

- quelques fois sans contexte particulier, et on peut évoquer un conflit neuro-ligamentaire sur le nerf pudendal avec un véritable syndrome canalaire du petit bassin.

L'analyse neuro-physiologique de toutes ces circonstances va nous permettre de mieux comprendre la physiopathologie de la douleur pelvienne chronique chez l'homme.

\section{PHYSIOPATHOLOGIE DE LA DOULEUR PELVIENNE CHRONIQUE}

\section{Quelle est la conséquence neurophysiologique d'une stimulation nociceptive ?}

N'importe quelle stimulation nociceptive provenant de n'importe quelle partie de l'organisme, entre autre à partir du pelvis, quelle soit d'origine post-traumatique, post opératoire, post-infectieuse ou autre, entraîne toujours les mêmes cascades neurophysiologiques [4].

Les messages nociceptifs sont véhiculés dans les nerfs par les fibres amyéliniques ou peu myélinisées $A d$ et $C$ vers la corne postérieure de la moelle épinière par la racine postérieure où siège le premier neurone (protoneurone). II n'assure qu'un rôle de transmission grâce aux substances algogènes libérées par la stimulation nociceptive. Les principales substances algogènes sont la bradykinine, la sérotonine, l'histamine, la substance $P$, le monoxyde d'azote, le facteur de croissance (NGF) et les prostaglandines. Elles interviennent dans l'inflammation neurogène 
induisant une dégranulation des mastocytes et une vasodilatation créant ainsi une véritable boucle d'entretien et une chronicité de la douleur.

Au niveau médullaire siège le deuxième neurone (deutoneurone), localisation de la mémoire de la douleur grâce à la sensibilisation centrale. En effet, d'une part il y a une rétroaction sur la libération périphérique des substances algogènes (réflexe d'axone entretenant la douleur), mais aussi l'arrivée des messages nociceptifs au niveau médullaire provoque une modification de la plasticité neuronale grâce à l'action entre autre du glutamate sur les récepteurs récepteurs mu, NDA et CCK (stigmate de la mémorisation douloureuse). Enfin ce message nociceptif arrive au niveau du cortex cérébral (aires S1 S2) pour être reconnu au niveau de l'intensité et de la localisation et surtout pour provoquer des réactions diverses (motrices, fonctionnelles, émotionnelles ...). Au niveau pelvien cela peut se traduire par la douleur chronique, une hyperalgésie et des dysfonctions organiques.

Cette cascade neurophysiologique à partir d'un stimulus nociceptif est résumée sur la Figure 1.

\section{Quelles sont les voies de la douleur au niveau pel- vien?}

L'innervation est double : somatique et végétative (ortho et para-sympathique).

a) L'innervation somatique est assurèe par le plexus sacré et le plexus honteux $[6,21]$.

La branche terminale du tronc lombo-sacré est le grand sciatique destiné aux membres inférieurs.

Le nerf pudendal (Figure 2) est issu de $\mathrm{S} 3$ et plus rarement de S2 et S4. II quitte la partie pré-sacrale pour se placer en dedans du tronc sciatique. II pénètre la région glutéale sous le muscle pyramidal et contourne la terminaison du ligament avant son insertion sur l'épine sciatique. II rentre donc en rapport avec la face ventrale du ligament sacrotubéral, puis gagne le pelvis via la petite ouverture sciatique dans un dédoublement de l'aponévrose du muscle obturateur interne appelé canal d'Alcock. Les branches terminales du nerf pudendal sont le nerf rectal inférieur à destinée anale, le rameau périnéal destiné à la peau du périnée et aux muscles ischio-caverneux, le nerf sensitif dorsal de la verge et du clitoris. Ainsi on peut décrire trois segments du trajet pudendal : une région pré-sacrale, un trajet glutéal dans la partie médiane du canal piriforme et sa division dans le canal d'Alcock.

Le trajet du nerf est tel qu'il est soumis à deux sortes de conflit :

- le premier dans le canal sous piriforme où il est pris dans une pince ligamentaire entre le ligament sacro-épineux et le ligament sacro-tubéral. Le muscle pyramidal peut également représenter un conflit qui intéresse en plus du nerf pudendal le nerf sciatique situé plus latéralement.

- la deuxième zone de conflit potentiel est dans le canal
d'Alcock où le nerf est plaqué dans un dédoublement aponévrotique où il subit des pressions du processus falciforme et l'ascension de la graisse ischio-rectale en position assise.

D'autres nerfs sont impliqués dans l'innervation du pelvis : le nerf ilio-inguinal et ilio-hypogastrique (L1), le nerf génitofémoral (L1 L2) et le nerf obturateur (L2 L3 L4).

La somatotopie du pelvis et du périnée est donc complexe avec intrication des racines lombaires et des racines sacrées. Elle est résumée sur la Figure 3.

b) L'innervation végétative est assurée par le plexus hypogastrique supérieur juste en dessous de la bifurcation iliaque. Après 5 ou $6 \mathrm{~cm}$ de trajet, le plexus hypogastrique se divise en 2 branches appelées nerf hypogastrique ou plexus hypogastrique inférieur. Celui-ci se glisse entre le sacrum et la lame pré-sacrée latéralement, se dissociant en plusieurs branches assurant l'innervation viscérale des organes du pelvis (vessie, prostate ...). II existe des anastomoses de chaque côté avec la chaîne sympathique lombaire, avec le sympathique sacré et avec le nerf pudendal par l'intermédiaire des nerfs érecteurs d'Eckhard. Ces nerfs érecteurs d'Eckhard contiennent essentiellement des fibres parasympathiques. Ainsi de façon latérale, au niveau des lames sacro-recto-génito-pubiennes, il existe une triple innervation ortho-sympathique, para-symphatique et somatique. Le contingent para-sympathique provient de D10, L1 et le contingent para-sympathique de la moëlle sacrée $S 2$, S3 et S4.

En ce qui concerne le testicule, l'innervation provient de deux systèmes végétatifs :

- spermatique naissant du plexus péri-aortique et anastomosé au ganglion aortico-rénal, il suit l'artère spermatique jusqu'à sa terminaison ;

- déférentiel issu du ganglion hypogastrique supérieur, il constitue le nerf du déférent qui suit le canal depuis la vésicule séminale jusqu'à la queue de l'épididyme. La peau scrotale est par contre innervée par le rameau périnéal du nerf pudendal.

L'organisation de l'innervation du pelvis est résumée sur la Figure 4.

\section{Quelles sont les conséquences d'une stimulation nociceptive post-opératoire sur le pelvis ?}

Zermann et al. en 1998 [32] ont publié une étude sur les conséquences de la chirurgie pelvienne. Ils ont montré qu'il se produisait un embrasement neurologique et une modification de la plasticité neuronale pouvant aboutir à une douleur chronique et à des dysfonctions vésicales résiduelles. Le blocage du systéme nerveux central avant la chirurgie soit par des anesthésiques locaux ou par un protocole d'analgésie préventive peut éviter cet emballement neurophysiologique et ainsi en éviter ses conséquences. Les sujets à risque tels que les patients présentant déjà une douleur chronique ou des troubles vésico-sphinctériens doivent être identifiés avant toute chirurgie pelvienne pour tenter d'éviter cet embrasement neurologique [32]. 


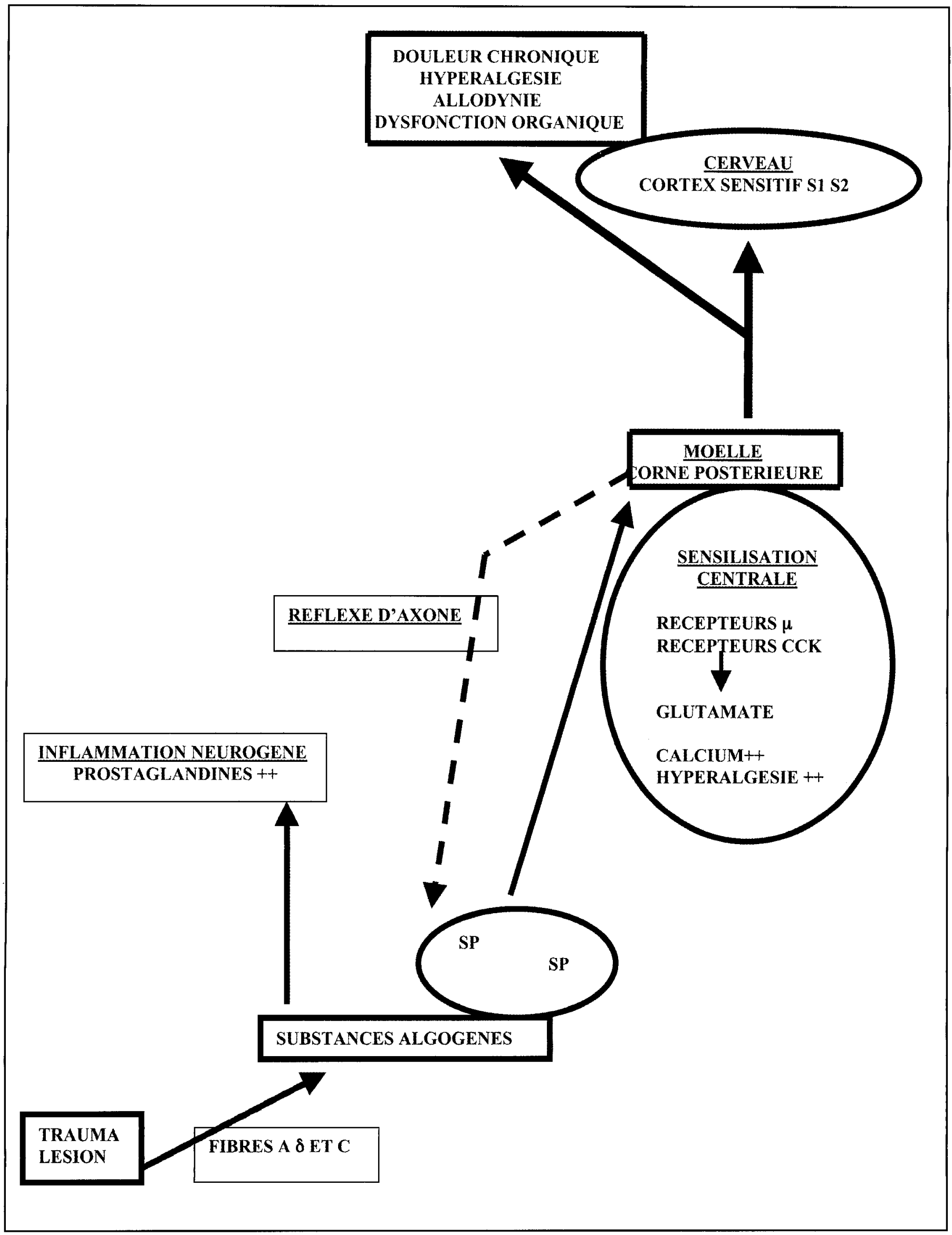

Figure 1 : Physiopathologie de la douleur. 


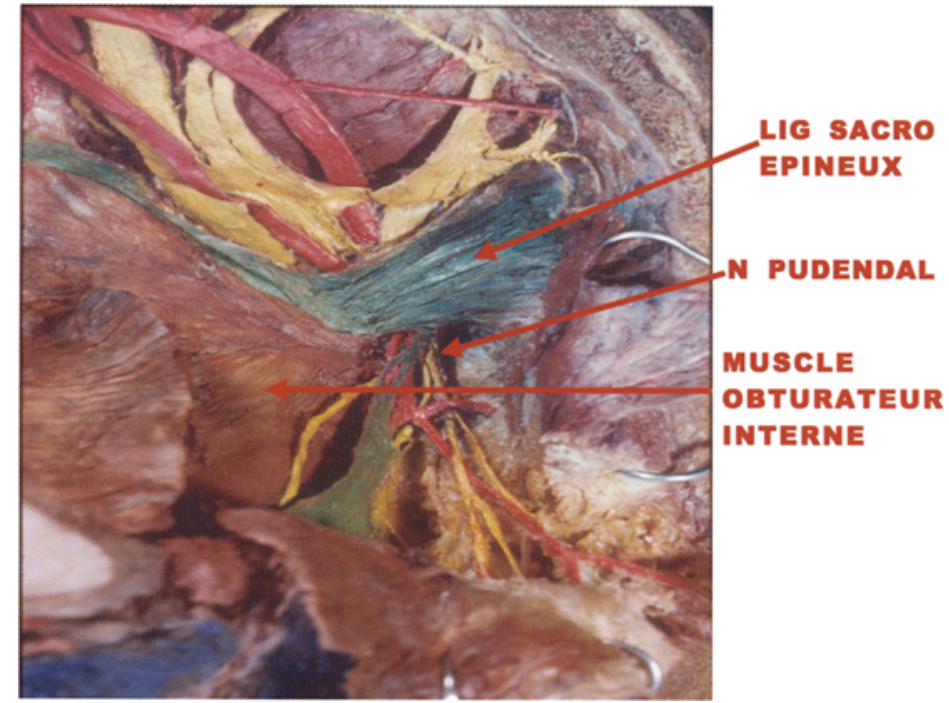

Figure 2 : Anatomie du nerf pudendal.
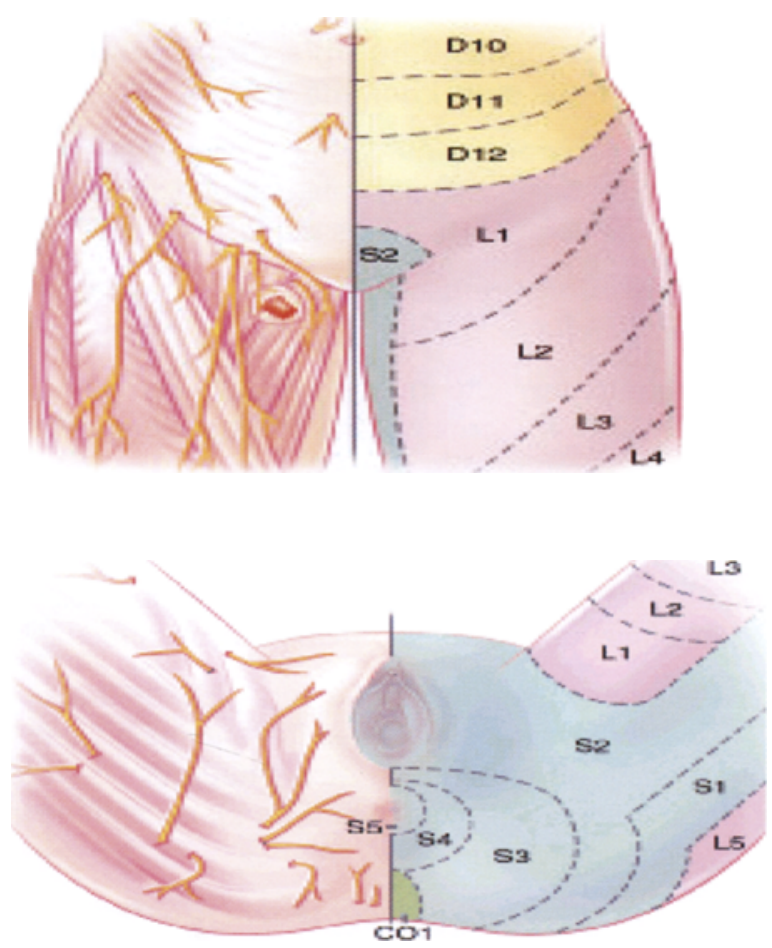

Figure 3 : Somatotopie du pelvis.

D10-L1

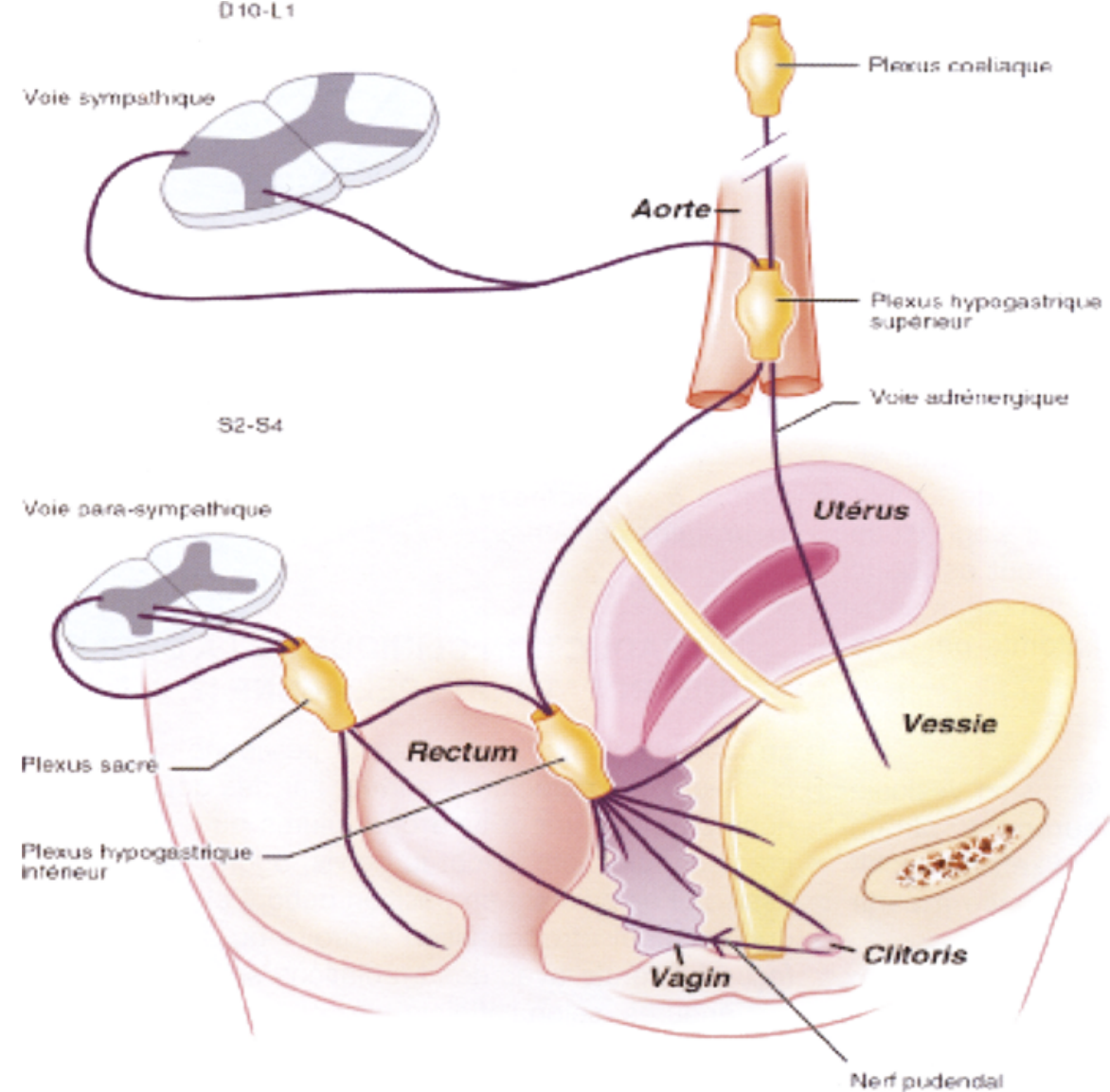

Figure 4 : Innervation du pelvis. 
4. Quelles sont les données récentes de la littérature sur la physiopathologie de la douleur pelvienne chronique chez l'homme?

On va séparer les études concernant spécifiquement la cystite chronique, la prostatite chronique, les douleurs testiculaires chroniques et des études plus générales.

\section{a) Les publications concernant la cystite chronique et intersticielle}

De nombreux auteurs Jasmin [12], Zermann [33], Steers [25], Dale [9], Zermann [34] et Wesselmann [29, 30] insistent sur l'importance de l'inflammation neurogène dans la genése de ces syndromes douloureux pelviens chroniques avec une hyperalgésie secondaire toujours présente et une implication certaine de neurotransmetteurs facilitateurs tels que la substance $P$ et l'hormone de croissance (NGF).

Les traitements proposés ne sont plus les traitements urologiques classiques mais des traitements à visée neurogène comme le gabapentin et les neuroleptiques avec une amélioration stastistique des symptômes comme la douleur chronique pelvienne.

\section{b) Les publications concernant la prostatite chronique}

Les auteurs suivants, Zermann [32], Mehik [17], Maake [16], et Potts [20] retrouvent également un rôle prédominant de l'inflammation neurogène dans l'explication neurophysiologique des prostatites chroniques rebelles aux traitements classiques avec également une sensibilisation centrale majeure par embrasement neurologique expliquant la douleur chronique séquellaire. Pour appuyer encore cette hypothèse neurogène Yang [31] découvre des anomalies neurogènes majeures dans les métamères S2 S3 au thermotest chez des patients souffrant de douleurs pelviennes chroniques rebelles avec des symptômes de type prostatite chronique. D'autres mécanismes neurophysiologiques ont été évoqués comme la médiation par les cytokines, donc une piste immunologique, par Jang [11].

\section{c) Les publications concernant la douleur testiculaire chronique rebelle}

Elle représente entre 5 à $10 \%$ des consultations en urologie. Si le bilan urologique est négatif (absence de tumeur ou d'infection), certains auteurs pensent qu'il s'agit d'une douleur de type végétative [28]. Le traitement est donc médical à visée neurogène, infiltratif (bloc du cordon spermatique) et parfois chirurgical (orchidectomie par voie inguinale).

\section{d) Les publications plus générales}

- Castroman en 2002 montre que la distension vésicale provoquée entraîne une réponse neuronale spinale avec activation des récepteurs NMDA. La kétamine inhibe cette activation avec un effet dose-réponse à partir de $1 \mathrm{mg} / \mathrm{kg}$ jusqu'à $10 \mathrm{mg} / \mathrm{kg}$ [7].

- Einsenberg en 2003 montre que la sensibilisation centrale secondaire est tout à fait identique qu'il s'agisse d'une cystite chronique ou d'une prostatite chronique [10].
- Levander en 2003 va plus loin et pense que l'inflammation neurogène secondaire est identique dans de nombreux syndromes douloureux chroniques comme la cystite chronique, le colon irritable ou la fibromyalgie et que par conséquent les bases thérapeutiques sont identiques [14].

Et en l'absence de toute les situations précédentes, sans notion de traumatisme grave, d'infection ou d'intervention chirurgicale pelvienne, ce qui correspond à un certain nombre de patients, on peut évoquer un syndrome canalaire sur le nerf pudendal. En effet, nous avons vu qu'il existait deux zones de conflit sur le nerf pudendal (conflit glutéal et au niveau du canal d'Alcock). Cette entité clinique à été décrite dés 1991 par Amarenco [2] puis par Bensignor et Robert en 1993 [5, 21] sous le nom de syndrome du canal d'Alcock ou névralgie périnéale s'exprimant comme une douleur de type neuropathique.

Au final, quelque soit les multiples circonstances d'apparition habituelles et même parfois sans étiologie particulière, la douleur pelvienne chronique chez l'homme se présente souvent sous la forme d'une douleur de type neuropathique chronique dont la physiopathologie est résumée sur la Figure 5.

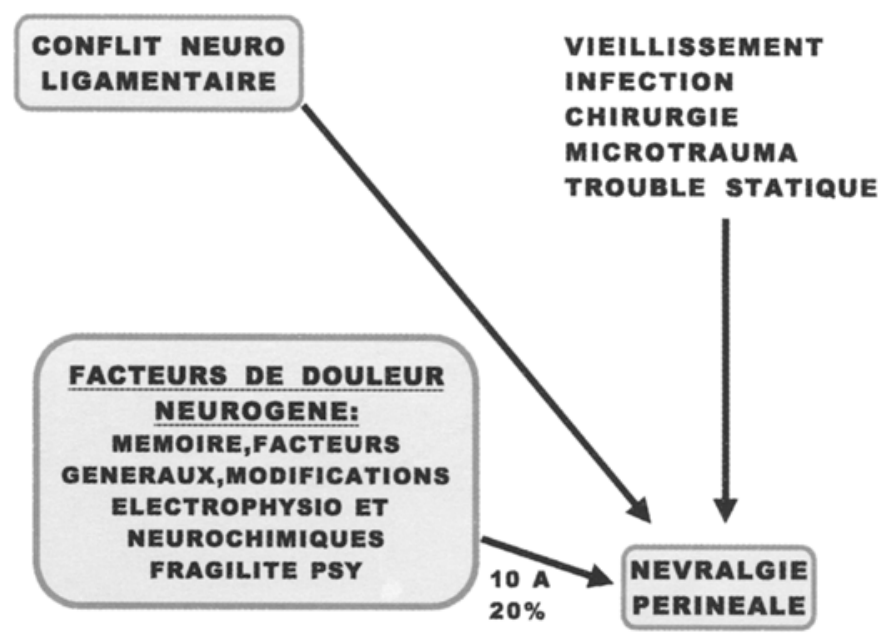

Figure 5 : Hypothèse physiopathologique de la douleur neurogène pelvienne.

\section{TRADUCTION CLINIQUE DE LA DOULEUR NEUROPATHIQUE PELVIENNE [2, 3, 5, 28]}

Le tableau clinique de la douleur pelvienne neuropathique chronique est caractérisé surtout par des douleurs spontanées permanentes à type de brûlures ou de paresthésies irradiant volontiers à tout le pelvis, aggravées en position assise et se calmant en position debout et à la marche. La douleur peut être localisée à droite ou à gauche, ou de façon bilatérale avec des irradiations soit antérieures, soit postérieures, selon l'atteinte plus ou moins spécifique d'un rameau nerveux. La diminution des douleurs lors du décubitus ou de la station debout et leur augmentation en posi- 
tion assise, témoignent de la mise en tension du nerf pudendal dans son canal et évoquent donc soit un conflit sur le nerf, soit beaucoup plus fréquemment une fibrose étendue.

Compte-tenu de la triple innervation du pelvis, d'autres signes cliniques seront souvent associés à savoir des signes urinaires (pollakiurie, dysurie, besoins fréquents et urgents), des signes digestifs (dyschésie et constipation), des sensations de contractures musculaires (contracture du releveur de l'anus et de l'obturateur interne), des troubles vaso-moteurs avec sueurs et sensation de chaleur et de froid et enfin souvent une impuissance mixte.

L'examen neurologique général et neuropérinéal est souvent normal sans anomalies des réflexes du cône terminal. Au toucher rectal, on retrouve lors de la palpation appuyée sur le nerf pudendal, au niveau de l'épine ischiatique, une douleur élective reproduisant la douleur spontanée. Parfois, c'est un véritable signe de Tinel signant une atteinte du nerf pudendal. On recherchera également des signes neurogènes somatiques de type allodynie (douleur déclenchée par une stimulation qui normalement ne produit pas de douleur) ou anesthésie métamérique, des signes neurogènes végétatifs de type vaso-moteurs et aussi le retentissement musculaire de type contracture musulaire périnéale (releveur de l'anus et obturateur interne). La technique de l'examen clinique est résumée sur la Figure 6.

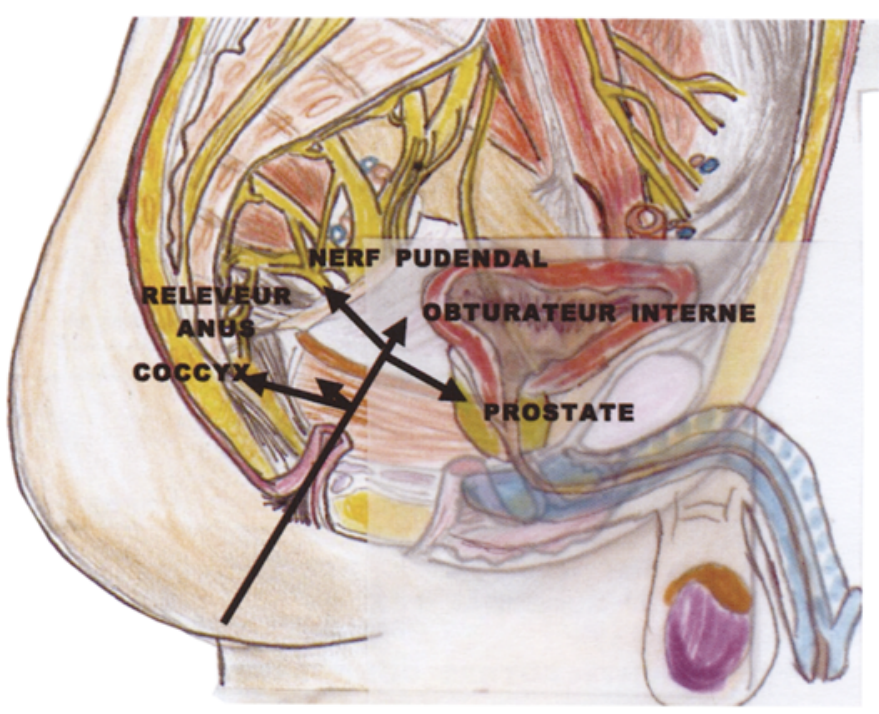

Figure 6 : Examen clinique du douloureux pelvien chronique.

L'exploration électromyographique comportant l'étude des latences sacrées, les potentiels évoqués somesthésiques et les latences distales motrices et sensitives du nerf pudendal est pratiquement systématique et permet d'objectiver en pratique les dénervations plus ou moins globales du pelvis mais aussi la névralgie pudendale (altérations des latences distales motrices et sensitives du nerf pudendal).
Les examens paracliniques sont habituellement normaux mais font partie du bilan systématique à savoir les radiographies osseuses, la scintigraphie osseuse, l'IRM pelvienne et l'IRM du cône terminal.

\section{TRAITEMENT DE LA DOULEUR PELVIENNE CHRONIQUE DE L'HOMME}

On va schématiquement isoler le traitement de la composante neuropathique et les traitement des signes cliniques associés.

1. Traitement de la composante neuropathique $[2,3,5$, $9,11,12,17,25,30,32-34]$

II repose sur un traitement médicamenteux, un traitement infiltratif, parfois chirurgical, et sur les techniques de neurostimulation.

\section{a) Le traitement médical classique}

C'est celui de toute douleur neurogène, à savoir les antidépresseurs tricycliques pour traiter la composante de brûlure continue et les anticonvulsivants pour traiter la composante fulgurante intermittente. Les antidépresseurs tricycliques les plus employés sont le Laroxyl ${ }^{\circledR}$ (amitriptyline) entre 25 et $100 \mathrm{mg} /$ jour et l'Anafranil ${ }^{\circledR}$ (clomipramine) entre 10 et $75 \mathrm{mg} / \mathrm{jour}$. Ce traitement peut provoquer une somnolence et doit être prescrit de façon progressive. Les traitements anticonvulsivants peuvent faire appel soit aux anciens anti-épileptiques (Rivotril ${ }^{\circledR}=$ clinazépam, Tegreto $^{\circledR}=$ carbamazépine), soit aux nouveaux anti-épileptiques (Neurontin ${ }^{\circledR}=$ gabapentin). Le Tegretol ${ }^{\circledR}$ (carbamazépine) doit être prescrit à dose progressive et croissante à partir de $200 \mathrm{mg}$ jusqu'à $1 \mathrm{~g} / \mathrm{jour}$, avec une surveillance enzymatique hépatique pour les fortes posologies. Le Rivotril ${ }^{\circledR}$ (clonazépam) peut être prescrit soit en comprimé soit en gouttes, à dose progressive et croissante également. Les nouveaux anti-épileptiques comme le Neurontin ${ }^{\circledR}$ (gabapentin) sont plus efficaces, provoquent moins d'effets secondaires et diminuent de façon plus importante les décharges douloureuses paroxystiques et l'allodynie. On prescrira donc le Neurontin ${ }^{\circledR}$ (gabapentin) à dose progressive et croissante à partir de $300 \mathrm{mg}$ jusqu'à 1600 $\mathrm{mg} / \mathrm{jour}$. Les anxiolytiques, les autres antidépresseurs et les neuroleptiques n'ont pas d'indication spécifique et ne traitent que l'anxiété ou la dépression réactionnelle. Les antalgiques des trois paliers de l'OMS ne traitent pas la douleur neuropathique aux posologies habituelles et n'ont donc pas de place spécifique dans le traitement de cette douleur hormis peut être pour des posologies fortes, notamment pour la morphine comme en témoignent les études actuelles sur l'efficacité de la morphine dans les douleurs neurogènes.

D'autres thérapeutiques peuvent être proposées : les anesthésiques locaux en injection intra-veineuse $(5 \mathrm{mg} / \mathrm{kg}$ de Lidocaine) et les anti-NMDA comme la Kétamine. La Kétamine a prouvé son efficacité dans des douleurs viscérales pelviennes chroniques à la posologie de 1,3 et 10 $\mathrm{mg} / \mathrm{kg} / \mathrm{jour}$ [7]. En pratique on peut proposer des injections intra-veineuses de Kétamine à la posologie de 0,6 mg/kg/j 
pendant 3 à 5 jours puis 2 fois par semaine pendant plusieurs mois. Ces protocoles sont en évaluation actuellement.

\section{b) Le traitement infiltratif.}

Diverses techniques d'infiltration du nerf pudendal ont été proposées (bloc transvaginal, bloc analgésique au niveau du canal d'Alcock, bloc transglutéal sous contrôle radiologique et une technique proximale personnelle). Seule l'infiltration proximale sera décrite (Figure 7 ).

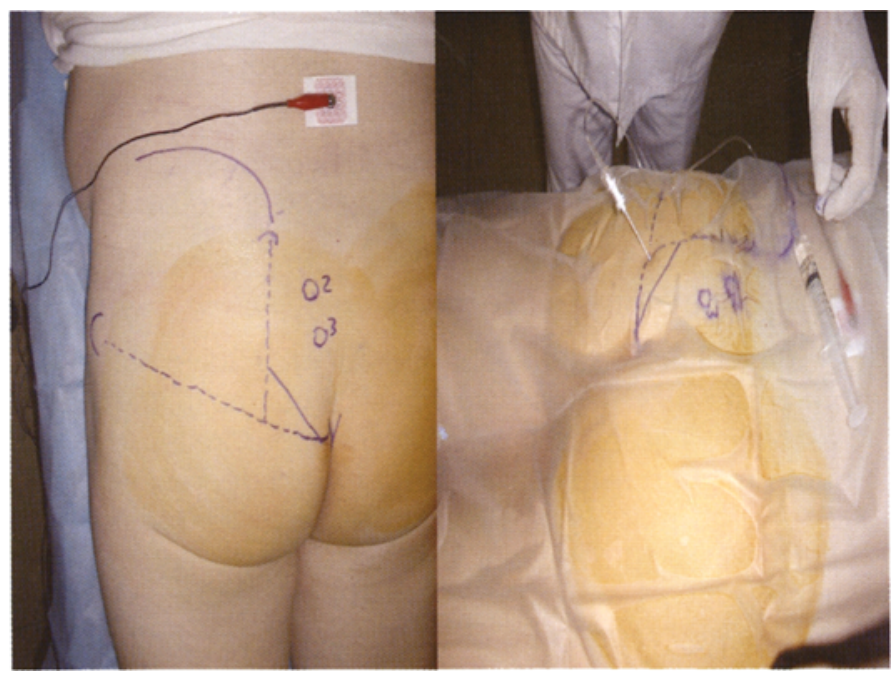

Figure 7 : Infiltration analgésique du nerf pudendal.

II s'agit d'une technique personnelle mise au point à partir de travaux anatomiques. Cette technique ne nécessite aucune infrastructure et est réalisée sans contrôle radiologique, ni scanographique. Le patient est placé à plat ventre et les injections sont réalisées au niveau de l'intersection de deux lignes : la ligne horizontale relie le grand trochanter et l'articulation sacro-coccygienne et la ligne verticale passe par l'épine iliaque postéro-supérieure. L'inclinaison de l'aiguille est de $30^{\circ}$, en direction du 2ème trou sacré. Sous neurostimulateur, on déclenche des paresthésies au niveau périnéal, reproduisant souvent la douleur et une injection de $80 \mathrm{mg}$ de corticoide retard associé à $4 \mathrm{ml}$ de Naropein ${ }^{\circledR} 7,5 \%$ (ropivacaine) est réalisée de façon uni ou bilatérale. Cette technique permet une infiltration très proximale au niveau du nerf, au dessus du ligament sacro-épineux et a donc une véritable valeur de test puisqu'elle peut améliorer non seulement un conflit sous-jacent, mais également une fibrose étendue sur le nerf. Une série de deux à trois blocs peut alors être réalisée avec une amélioration durable.

Une infiltration peut être réalisée au niveau du cordon spermatique, le plus haut possible avec une association de 2 à $3 \mathrm{ml}$ d'anesthésique local et $80 \mathrm{mg}$ de corticoïdes retards. Cette technique est proposée pour traiter les douleurs testiculaires chroniques d'abord de façon diagnostique puis thérapeutique. En effet une amélioration après le 1 er bloc peut nécessiter plusieurs autres infiltrations pour avoir un effet durable. Par contre un échec d'une première infiltration ne nécessite pas a priori d'autres infiltrations.

\section{c) Le traitement chirurgical}

II est rare et ne concerne que la névralgie pudendale par conflit neuroligamentaire rebelle aux infiltrations. II a été mis au point par Robert et al. en 1993 (60 à 70\% d'amélioration) [21].

\section{d) La neurostimulation}

Comme dans toute douleur neurologique, les techniques de neurostimulation peuvent être utilisées $[2,3,5,21]$. La neurostimulation transcutanée au niveau des métamères S2 ou S3 est difficile à mettre en place et donc elle est peu utilisée. La neuromodulation des racines sacrées est surtout préconisée dans le traitement de certains troubles mictionnels mais on ne dispose pas d'études sur son efficacité dans le traitement de la douleur pelvienne chronique. Par contre, une étude de Van Balken en 2003 montre l'efficacité de la neurostimulation tibiale postérieure sur les douleurs pelviennes chroniques à prédominance neurogène [27].

2. Traitement des signes cliniques associés $[1,8,19$, $23,26]$

II est indispensable de traiter de façon simultanée les troubles vésico-sphinctériens, les troubles recto-sphinctériens, l'impuissance et le retentissement psychologique de la douleur pelvienne chronique avec toutes les thérapies classiquement proposées.

En conclusion, on peut proposer la stratégie thérapeutique suivante pour les douleurs pelviennes chroniques rebelles (Figure 8) :
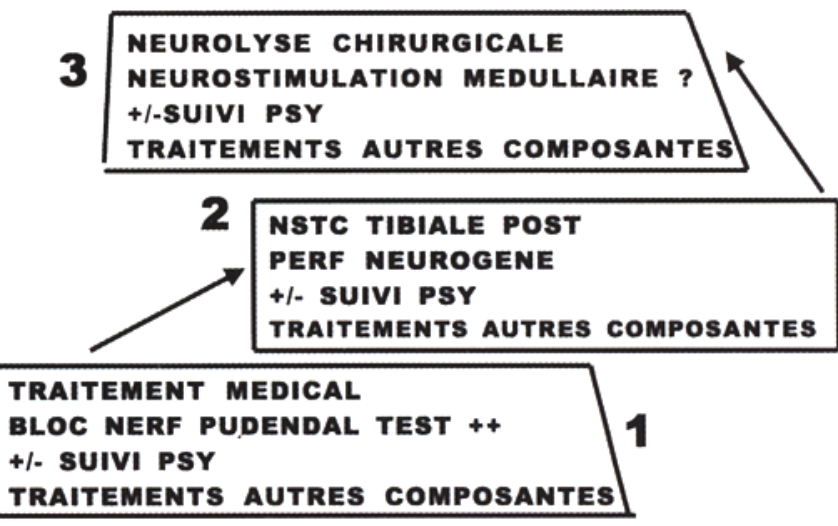

EVALUATION +++

Figure 8 : Les trois paliers du traitement de la douleur neurogène pelvienne chronique.

- en premier niveau, une association d'un traitement médical guidé sur les caractéristiques de la douleur neurogène, d'une infiltration du nerf pudendal ou d'un autre nerf somatique du pelvis selon la somatotopie de la douleur, et d'un traitement des signes cliniques associés et des autres composantes douloureuses en particulier psychologique ;

- en deuxième niveau, une association d'un traitement à visée neurogène par voie intraveineuse, un protocole de 
neurostimulation tibiale postérieure et un traitement des signes cliniques associés et des autres composantes de la douleur ;

- en troisième niveau, des traitements plus lourds et plus hypothétiques comme les techniques de neurostimulation médullaire ou la chirurgie de décompression du nerf pudendal.

\section{CONCLUSION}

Les connaissances physiopathologiques concernant la douleur pelvienne chronique ont été longtemps imprécises et par conséquent sources de nombreux échecs thérapeutiques. Actuellement elles sont mieux connues grâce à une littérature de plus en plus abondante, et le mécanisme neuropathique semble devenir prédominant, en particulier dans les prostatites chroniques. Les progrès pharmacologiques vont de pair et on dispose actuellement de thérapeutiques à visée neurogène assez efficaces comme le gabapentin ou certaines techniques d'infiltration périnerveuse ou de neurostimulation. L'intrication de la douleur avec les signes recto-sphinctériens, vésico-sphinctériens, une altération de la qualité de vie et un retentissement psychologique souvent majeur montrent de toute évidence la nécessité d'associer plusieurs thérapeutiques (traitement de la douleur et des signes cliniques associés) avec une réévaluation régulière et une prise en charge globale et pluridisciplinaire.

\section{REFERENCES}

1. ALAGIRI M., CHOTTINER S., RATNER V. et al. : Interstitial cystisis : unexplained associations with chronic disease and pain syndromes. Urology, 1997, $49: 52-57$.

2. AMARENCO G., LECOCCQUEN-AMARENCO A., KERDRAON J. et al. : Les névralgies périnéales. La Presse Médicale, 1991, $20: 71-74$.

3. BAUDE C., GALAUP J.P., SUTET P. et al. : Douleur pelvi-périnéale chronique rebelle. J. Méd. Lyon, 2001, 8, 1529 : 11-15.

4. BAUDE C. : Bases neuro-physiologiques de le douleur. In : De Broca A. ed. Douleur, Soins Palliatifs, Deuil. Paris, Masson, $2002: 3-14$.

5. BENSIGNOR M., LABAT J.J., ROBERT R. et al. : Douleur périnéale et souffrance des nerfs honteux internes. Cahier d'Anesthésiologie, 1993, 41, $2: 22-27$.

6. BOSSY J. : Anatomie clinique. Paris, Springer Verlag, 1990.

7. CASTROMAN P.J., NESS T.J. : Ketamine, an Methyl-D-aspartate receptor antagonist, inhibits the spinal neuronal responses to distension of the rat urinary bladder. Anesthesiology, 2002, $96: 1410-1419$.

8. CURTIS J., NICKEL P. : Effective office management of chronic prostatitis. Urol. Clin. North Am., 1998, 25 : 677-683.

9. DALE E., BJORLING K., ZUN-YI-WANG K. : Oestrogen and neuro inflammation. Urology, 2001, $57:$ 40-46.

10. EISENBERG E.R., MOLDWIN R.M. : Etiology : where does prostatitis and interstitial cystisis begin ? World J. Urol., 2003, $21: 64-69$.

11. JANG T.L., SCHAEFFER A.J. : The role of cytokines in prostatitis. World J. Urol., 2003, $21: 95-99$.
12. JASMIN L., JANNI G., MANZ H.J. et al. : Activation of CNS circuits producing a neurogenic cystisis : evidence for centrally induced peripherical inflammation. J. Neurosci., 1998, 18 : 10016-10029.

13. KRIEGER J.N., ROSS S.O., RILEY D.E. : Chronic prostatitis : epidemiology and role of infection. Urology, 2002, $60: 8-12$.

14. LEVANDER H. : Sensory sensitization, part 2 : Pathophysiology in dysfunctional disorders. Understanding the inner life of the nerve pathways may explain hitherto unexplainable symptoms. Lakartidningen, 2003, $100: 1618-1619$.

15. LUMMUS W.E., THOMPSON I. : Prostatitis. Emerg. Med. Clin North Am., 2001, $19: 691-707$.

16. MAAKE C., JOHN H. : Prostatitis versus pelvic pain syndrome: immunologic studies. Curr. Urol. Rep., 2003, 4 : 327-334.

17. MEHIK A., LESKINEN M.J., HELLSTROM P. : Mechanisms of pain in chronic pelvic pain syndrome influence of prostatic inflammation. World J. Urol., 2003, 21 : 90-94.

18. NEWSOME G. : Intertitial cystitis. J. Am. Acad. Nurse Pract., 2003, $15: 64-71$.

19. PEEKER R., FALL M. : Treatment guidelines for classic and non ulcer interstitial cystitis. Int. Urogynecol. J. Pelvic Floor Dysfunct., 2000, $11: 23-33$.

20. POTTS J.M. : Chronic pelvic pain syndrome : a non prostatocentric perspective. World J. Urol., 2003, 21 : 54-56.

21. ROBERT R., LABAT J.J., BENSIGNOR M. et al. : Bases anatomiques de la chirurgie du nerf pudendal : conséquences thérapeutiques dans certaines algies périnéales. Lyon Chir., $1993,89 / 3: 115-137$.

22. ROBERTS R.O., JACOBSON D.J., GIRMAN C.J. et al. : Prevalence of prostatitis-ike symptoms in a community based cohort of older man. J. Urol., 2002, $168: 2467-2471$.

23. ROMAN S., BELAISE C., MARTIN J. et al. : Childhood abuse and later medical disorders in women : an epidemiological study, Psychoter. Psychosom., 2002, 71 : 141-150.

24. SCHAEFFER A.J., LANDISJ.R., KNAUSS K.J. et al. : Demographic and clinical characteristics of men with chronic prostatitis : the national institutes of health chronic prostatitis cohort study. J. Urol., 2002, $168:$ 593-598

25. STEERS W.D. : Interstitial cystisis : past and future. Urology, 2001, $57: 101-102$.

26. STERVERMER J.J., EASLEY S.K. : Treatment of prostatitis, Am. Fam. Physician, 2000, 61: 10-17.

27. VAN BALKEN M.R., VANDONINCK V., MESSELINK B.J. et al.: Percutaneous tibial nerve stimulation as neuromodulative treatment of chronic pelvic pain. Eur. Urol., 2003 : 43 : 158163.

28. WESSELMANN U., BURNETT A.L., HEINBERG L.J. : The urogenital and rectal pain syndromes. Pain, 1997, 73 : 269294.

29. WESSELMANN U., CZAKANSKI P.P. : Pelvic pain : a chronic visceral pain syndrom. Curr. Pain Headache Rep., 2001, 5 : 13-19.

30. WESSELMANN U. : Interstitial cystisis : a chronic visceral pain syndrom. Urology, 2001, $57: 32-39$.

31. YANG C.C., LEE J.K., KROMM B.G. et al. : Pain sensitization in male chronic pelvic pain : why are symptoms so difficult to treat ? J. Urol., 2003, $170: 823-826$.

32. ZERMANN D.H., ISHIGOOKA M., DOGGWEILER R. et al. : Postoperative chronic pain and bladder dysfunction : windup and neuronal plasticity-do we need a more neurological approach in pelvic surgery ? J. Urol., 1998, 160, 102-105. 
33. ZERMANN D.H., ISHIGOOKA M., DOGGWEILER R. et al. Neurourological insights into the genitourinary pain in men. $J$ Urol., 1999, $161:$ 903-908.

34. ZERMANN D.H., ISHIGOOKA M., DOGGWEILER-WIYGUL R. : The male chronic pelvic pain syndrome. World J. Urol., 2001, $19: 173-179$.

Communication au $X X^{\circ}$ Congrès de la Société d'Andrologie de Langue Française, Orléans, 11-13 décembre 2003.

Manuscrit reçu : décembre 2003 ; accepté : janvier 2004.

\section{ABSTRACT}

Pathophysiology and treatment of chronic pelvic pain in men

\section{Christian BAUDE}

Chronic pelvic pain, in young men or elderly men, has always been a challenge to the medical profession, raising problems of assessment and management.

Chronic pelvic pain has a high prevalence, which is underestimated as indicated by the following figures: $4 \%$ to $8 \%$ of patients consulting chronic pain centres, $15 \%$ of patients consulting a urologist for symptoms of chronic prostatitis with alteration of quality of life, 70,000 cases of chronic cystitis per year in the USA.

The circumstances of onset are multiple: postoperative, after minor or major trauma or postinfectious, sometimes without any particular aetiology and often in a multifactorial context.

The pathophysiology is therefore vague and poorly elucidated, as only about $5 \%$ of cases of chronic prostatitis have a bacterial cause. However, any form of stimulation activates pain pathways with neurogenic inflammation followed by central sensitization and modification of neuronal plasticity, and finally chronic refractory pain with organic dysfunction.

This mechanism is currently proposed in numerous publications concerning postoperative chronic pelvic pain and refractory cystitis and chronic prostatitis. The pathophysiology of these types of pain is probably therefore neurogenic. In the absence of stimulation, a pudendal nerve tunnel syndrome can be suggested.

The treatment of chronic pelvic pain in men can be considered in the following way: aetiological treatment whenever possible, neurogenic medical treatment (tricyclic antidepressants for continuous pain, anticonvulsants for intermittent pain, NMDA receptor antagonists in the case of failure), treatment of organic dysfunction, pudendal nerve analgesic block in the case of suspected tunnel syndrome and global treatment of patient with impaired quality of life.

In conclusion, a better pathophysiological approach to these forms of chronic pelvic pain can improve these difficult patients.

Key-Words: chronic pelvic pain, chronic cystitis, chronic prostatitis, pudendal nerve 